\title{
Analysis of Nash Equilibria in Energy Markets with Large Populations of Price-Responsive Flexible Appliances
}

\author{
Antonio De Paola, David Angeli and Goran Strbac
}

\begin{abstract}
This paper deals with flexible electrical devices that, on the basis of a broadcast price signal, schedule their individual power consumption in order to minimize their energy cost. If the devices population is sufficiently large to be described as a continuum, it is possible to provide necessary and sufficient conditions for the existence of a Nash equilibrium in the energy market. This is done by comparing two functions which characterize, respectively, the valley capacity of the inflexible demand and the global properties of the appliances population. The equilibrium conditions, which do not require any iterative procedure to be applied, are finally tested in simulations.
\end{abstract}

\section{INTRODUCTION}

Following recent trends in the evolution of power systems, it is expected that in the near future a considerable number of flexible devices (e.g. "smart appliances" and electric vehicles) will participate in system operation by scheduling their power consumption during the day. The potential advantages of this scenario, such as reduction of costs for the customers and flattening of demand profile, have been studied in detail [1], [2]. On the other hand, significant challenges arise if one considers the management of these devices. In particular, in order to avoid synchronicity phenomena characterized by the whole population operating at low prices and shifting the peak demand, it is important to account for the energy price variations introduced by the aggregate power consumption of the appliances. This problem can be approached with centralized techniques, solving a global optimization which accounts for objectives and constraints of customers and generators, as proposed in [3] and [4]. Given the potential high number of devices, privacy concerns and the traditional tendency of private customers to fully control their operation strategy, distributed approaches have also been considered. For example, [5] adopts the concept of congestion pricing and proposes an adaptive strategy based on price feedbacks. A two-level iterative process with Lagrangian relaxation is considered in [6] to integrate flexible demand in the energy market while [7] applies game theory to determine the power profile of the devices as the best response to certain tariffs. This approach is extended in [8] approximating the population size as infinite, neglecting the contribution of the single device and modelling the problem as a mean field game. Interesting techniques have been proposed for the specific scenario of electric vehicles integration in the grid: for example in [9] the charge profile of the vehicles is calculated

A. De Paola, D. Angeli and G. Strbac are with the Dept. of Electrical and Electronic Engineering of Imperial College London, UK. Email: ad57090imperial.ac.uk. The work is partially supported by FP7 grant 283145 "ClusterDesign". through an iterative strategy which converges to equilibrium by penalizing variations with respect to the previous iteration.

A game theory framework is also adopted in this paper, modelling the devices as competing agents that aim to operate at minimum cost. They do so by scheduling their power consumption on the basis of a broadcast demand/price signal received by some central entity. We are interested in determining the existence of Nash equilibria in the system. In other words, we want to understand when the power consumption profiles of the devices, formulated on the basis of the broadcast signal, are optimal also when the price of the resulting aggregate demand is considered. The main element of novelty of this work is that the proposed conditions do not require any iteration and provide convergence to equilibrium in one step. In particular, they are obtained comparing two different functions: a negotiable valley capacity which describes the capability of the system to absorb more power while preserving an equilibrium, and a power density of task durations which characterizes the global properties of the appliances population.

The rest of the paper is structured as follows: Section II describes the modelling choices for the devices and the energy market. The equilibrium conditions are introduced in Section III and tested in simulations in Section IV. Finally, conclusive remarks are presented in Section V.

\section{Modelling of Appliances And Energy Market}

The existence of Nash equilibria is investigated for energy markets with price-responsive appliances. Each appliance, at the beginning of the considered time interval $[0, T]$, broadcasts to a central entity (e.g. the system operator) the total energy $E_{t o t}$ that needs to consume and the minimum time $t_{\min }$ required to do so by operating at rated power $P_{r}$. These parameters can describe any kind of shiftable interruptible load, such as electric vehicles or electronics charging devices. If the number of appliances is sufficiently large to be described as a continuum, we can derive the unnormalized distribution $m$ of the parameters within the population. In particular, the integral $\int_{t_{1}}^{t_{2}} \int_{E_{1}}^{E_{2}} m(t, E) d E d t$ represents the number of devices for which $t_{1} \leq t_{\min } \leq t_{2}$ and $E_{1} \leq E_{t o t} \leq E_{2}$. For the purposes of the present work, the properties of the appliances population can be summarized by the function $f(t)$, which denotes the aggregate amount of energy required by the appliances with $t_{\min } \leq t$, and its derivative $f^{\prime}$. Denoting by $\mathscr{E}$ an interval which includes all broadcast values of $E_{t o t}$, they can be defined as:

$$
f(t):=\int_{0}^{t} \int_{\mathscr{E}} m(\tau, E) E d E d \tau \quad f^{\prime}(t):=\int_{\mathscr{E}} m(t, E) E d E
$$


Assumption 1: The derivative $f^{\prime}(t)=\int_{\mathscr{E}} m(t, E) E d E$ is assumed of compact support:

$$
\mathscr{F}=\operatorname{supp}\left(f^{\prime}\right)=\left[q_{\min }, q_{\max }\right]
$$

This can correspond to a scenario of heterogeneous appliances with different parameters $t_{\text {min }}$ and $E_{t o t}$ but can also represent the case of homogeneous devices which, at the beginning of the considered time interval $[0, T]$, are performing distinct tasks that require different amounts of time to be completed.

Regarding the modelling of the energy market, we consider an aggregate demand $D_{a}$ in the power system which has two distinct components: an inflexible power demand profile $D_{i}(\cdot)$, assumed to be known without uncertainties, and the flexible demand $D_{f}(\cdot)$ which represents the aggregate power consumption of the appliances population and will be defined later on. The energy price $p(t)$, at a given time instant $t$, is modelled as a strictly monotonically increasing function $\Pi$ of the current aggregate demand:

$$
p(t)=\Pi\left(D_{a}(t)\right)
$$

It is assumed that each appliance schedules in advance its power consumption $u$ according to a demand function $D(t)$ (or the corresponding price $\Pi(D(t)))$ received from the mentioned central entity. In particular, it minimizes the cost of completing the desired task within the time interval $[0, T]$ :

$$
\begin{array}{ll}
\min _{u(\cdot)} & \int_{0}^{T} \Pi(D(t)) \cdot u(t) d t \\
\text { s. t. } & 0 \leq u(t) \leq \frac{E_{\text {tot }}}{t_{\text {min }}} \\
& \int_{0}^{T} u(t) d t=E_{\text {tot }}
\end{array}
$$

One can verify that the solution to (3) corresponds to operate, at rated power $P_{r}=\frac{E_{t o t}}{t_{\min }}$, during the $t_{\min }$ hours with lowest broadcast demand $D$ (or equivalently with lowest price $\Pi(D)$ ). To provide a formal expression of the optimal power consumption profile, the following assumption is crucial:

Assumption 2: The broadcast demand is a continuous function $D:[0, T] \rightarrow[0,+\infty)$ with no level sets of positive measure. For any $d \in \operatorname{Im}(D)=\left[d_{\text {min }}, d_{\text {max }}\right]$, it holds:

$$
\mu(\{\tau \in[0, T]: D(\tau)=d\})=0
$$

where $\mu$ denotes the Lebesgue measure.

This assumption is not very restrictive and holds in general for typical profiles of inflexible demand, which will be considered later on when determining the equilibrium conditions. We now introduce the following quantity:

Definition 1: Given a broadcast demand profile $D:[0, T] \rightarrow[0,+\infty)$, it is possible to specify the associated function $Q_{D}:\left[d_{\min }, d_{\max }\right] \rightarrow[0, T]$, defined as:

$$
Q_{D}(d):=\mu(\{\tau \in[0, T]: D(\tau) \leq d\})
$$

The function $Q_{D}$ returns the measure of the sublevel sets of the demand $D$. Under Assumption 2, $Q_{D}$ is continuous, strictly monotone increasing and takes the following values at the endpoints of its domain:

$$
Q_{D}\left(d_{\min }\right)=0 \quad Q_{D}\left(d_{\max }\right)=T
$$

It can be shown that, as a result of these properties, the optimal power profile of each device is unique. In fact, for all values of $t_{\min }$, there exists a sublevel set of $D$ of measure $t_{\text {min }}$ that we denote by $\mathscr{S}_{D}\left(t_{\text {min }}\right)$ and define as follows:

$$
\mathscr{S}_{D}\left(t_{\text {min }}\right)=\left\{t \in[0, T]: Q_{D}(D(t)) \leq t_{\text {min }}\right\}
$$

Considering expression (5) and Assumption 2, it holds:

$$
D\left(t_{1}\right)<D\left(t_{2}\right) \quad \forall t_{1} \in \mathscr{S}_{D}\left(t_{\text {min }}\right) \forall t_{2} \in[0, T] \backslash \mathscr{S}_{D}\left(t_{\text {min }}\right)
$$

The optimal power consumption profile $u^{*}$ for devices with $t_{\text {min }}=s$ and $E_{t o t}=x$, when the demand $D$ is broadcast, can then be defined as:

$$
u^{*}(t, s, x)=\left\{\begin{array}{ccc}
\frac{x}{s} & \text { if } & t \in \mathscr{S}_{D}(s) \\
0 & \text { if } & t \notin \mathscr{S}_{D}(s)
\end{array}\right.
$$

As mentioned before, given that $\mu\left(\mathscr{S}_{D}(s)\right)=s$, the single device will operate at rated power $P_{r}=\frac{x}{s}$ during the $s$ hours characterized by lowest demand and price.

An alternative representation of the optimal power profile can be derived if one introduces the variable $q$, defined as the measure $Q_{D}(D(t))$ obtained evaluating (5) for $d=D(t)$. In this case the optimal power $\bar{u}^{*}$ of the individual device with $t_{\min }=s$ and $E_{\text {tot }}=x$ can be defined as follows:

$$
\bar{u}^{*}(q, s, x)=\left\{\begin{array}{lll}
\frac{x}{s} & \text { if } & q \leq s \\
0 & \text { if } & q>s
\end{array}\right.
$$

This notation can be extended to the aggregate power density $\bar{g}$ consumed by the devices with $t_{\min }=s$, as a function of $q$ :

$$
\bar{g}(q, s)=\int_{\mathscr{E}} \bar{u}^{*}(q, s, x) \cdot m(s, x) d x=\left\{\begin{array}{cc}
\frac{f^{\prime}(s)}{s} & \text { if } q \leq s \\
0 & \text { if } q>s
\end{array}\right.
$$

By taking the integral of $\bar{g}$ over the different minimum times $t_{m i n}$, we obtain the expression of the flexible demand $\bar{D}_{f}$ generated by the appliances population when $D$ is broadcast:

$$
\bar{D}_{f}(q)=\int_{0}^{T} \bar{g}(q, s) d s=\int_{q}^{T} \frac{f^{\prime}(s)}{s} d s
$$

Remark 1: The optimal power consumption profile $u^{*}$, the aggregate power density $g$ and the flexible demand $D_{f}$ as functions of time can always be obtained by evaluating the corresponding expressions in the $q$ variable at $q=Q_{D}(D(t))$ :

$$
\begin{gathered}
u^{*}(t, s, x)=\bar{u}^{*}\left(Q_{D}(D(t)), s, x\right) \\
g(t, s)=\bar{g}\left(Q_{D}(D(t)), s\right) \quad D_{f}(t)=\bar{D}_{f}\left(Q_{D}(D(t))\right)
\end{gathered}
$$

It is worth mentioning that, in the case of strictly increasing broadcast demand $D$, the values of the time variable $t$ and the measure $q$ will coincide, with $q=Q_{D}(D(t))=t$.

Once the flexible demand obtained for a certain broadcast $D$ has been determined, it is possible to calculate the corresponding aggregate profile $D_{a, D}$ as a function of time:

$$
D_{a, D}(t)=D_{i}(t)+\bar{D}_{f}\left(Q_{D}(D(t))\right)
$$


It is of particular interest the case $D=D_{i}$ (when the inflexible demand is broadcast) since in this scenario the corresponding aggregate $D_{a, D_{i}}$ depends only on the broadcast demand value $d=D_{i}(t)$ through the function $K(d)$ :

$$
D_{a, D_{i}}=K(d)=\left\{\begin{array}{llll}
d & \text { if } & Q_{D_{i}}(d)>q_{\max } \\
d+\int_{q_{\min }}^{q_{\max }} \frac{f^{\prime}(s)}{s} d s & \text { if } & Q_{D_{i}}(d)<q_{\min } \\
d+\int_{Q_{D_{i}}(d)}^{q_{\max }} \frac{f^{\prime}(s)}{s} d s & \text { if } & Q_{D_{i}}(d) \in \operatorname{supp}\left(f^{\prime}\right)
\end{array}\right.
$$

\section{NECESSARY AND SUFFICIENT CONDITIONS FOR NASH EQUiLIBRIUM}

It is desirable to understand under which conditions a Nash equilibrium is achieved in the energy market. In particular we want to determine, if they exist, the broadcast profiles $D$ such that the corresponding $u^{*}$ of all devices are optimal power schedules also for the resulting aggregate demand $D_{a, D}$. In other words all the appliances, which have optimized their power consumption considering the broadcast $D$, will have no unilateral interest in changing it in response to the variation of prices induced by considering the aggregate demand. The analysis of the equilibria will be carried out by comparing two different functions in the variable $q$ which are related to the chosen broadcast profile and to the properties of the appliances population.

Consider that high penetration of flexible demand has the potential of transforming valleys of the broadcast demand signal into peaks of the aggregated demand profile. In this case the decentralized power scheduling computed by the appliances is suboptimal and constantly susceptible to renegotiation in a way that forbids convergence to a well defined power consumption profile. For a given $D$, it is possible to quantify the amount of flexible demand that can be greedily allocated by the appliances while preserving the existence of a Nash equilibrium. We name this quantity the negotiable valley capacity, and define it as follows:

Definition 2: Given a broadcast demand profile $D:[0, T] \rightarrow[0,+\infty)$ which fulfils Assumption 2, we define the negotiable valley capacity as the function $\Lambda_{D}:[0, T] \rightarrow[0,+\infty)$ presented below:

$$
\Lambda_{D}(q): q \rightarrow \frac{d}{d q} Q_{D}^{-1}(q)
$$

Note that such definition does not depend on the energy price $\Pi$ and represents an indicator of the flexible power density that can be allocated in the valleys of the broadcast $D$.

In a similar way, it is possible to describe how a certain population of appliances will allocate their power consumption $u$ as a function of $q$, based on the distribution of the parameters $t_{\min }$ and $E_{\text {tot }}$. Introducing the notion of power density at $q$, the following definition can be provided:

Definition 3: For a given distribution $m$ of the appliances parameters, we define the power density of task durations as the function $\Lambda_{f}:\left[q_{\min }, q_{\max }\right] \rightarrow[0,+\infty)$ given below:

$$
\Lambda_{f}: q \rightarrow \frac{f^{\prime}(q)}{q}=\frac{\int_{\mathscr{E}} m(q, E) E d E}{q}
$$

Notice that $\Lambda_{f}(q)=\frac{f^{\prime}(q)}{q}$ is a density function which quantifies the total rated power of the appliances with equal parameter $q=t_{\text {min }}$. Therefore, given $q_{1}, q_{2} \in \operatorname{supp}\left(f^{\prime}\right)$, the integral $\int_{q_{1}}^{q_{2}} \Lambda_{f}(\tau) d \tau$ is equal to the aggregate rated power of the appliances with $t_{\text {min }} \in\left[q_{1}, q_{2}\right]$. It will be shown in the rest of this section how necessary and sufficient conditions for the existence of a Nash equilibrium can be provided by comparing the values of $\Lambda_{D_{i}}$ and $\Lambda_{f}$.

If one initially considers the problem in the time variable $t$, a Nash equilibrium exists if and only if the flexible demand scheduled on the basis of the a priori broadcast price signal $\Pi(D)$ is also optimal for the a posteriori price signal (viz. taking into account the sum $D_{a, D}$ of the inflexible and flexible demand). This is equivalent to impose that the following holds for all $E_{t o t}=x \in \mathscr{E}$ and $t_{\min }=s \in\left[q_{\min }, q_{\max }\right]$ :

$$
\begin{array}{ll}
\left.\min _{u(\cdot)} \int_{0}^{T} \Pi\left(D_{a, D}(t)\right) u(t) d t=\int_{0}^{T} \Pi\left(D_{a, D}(t)\right)\right) u^{*}(t, s, x) d t \\
\text { s. t. } & 0 \leq u(t) \leq \frac{x}{s} \\
& \int_{0}^{T} u(t) d t=x
\end{array}
$$

Remark 2: An alternative economic interpretation can be provided if, rather than analysing the population behaviour within a game theory framework, we consider all appliances as price-elastic individual consumers, viewing the exchanged power at each time instant as a commodity. The single device has no significant impact on the final energy price, implying that (16) characterizes a competitive equilibrium.

Given the optimality of the power profile $u^{*}$ for (3), its expression provided in (9) and the monotonicity of the price function $\Pi$, the following is verified for all $t_{\min } \in \operatorname{supp}\left(f^{\prime}\right)$ :

$$
D\left(t_{1}\right) \leq D\left(t_{2}\right) \quad \forall t_{1} \in \mathscr{S}_{D}\left(t_{\text {min }}\right) \quad \forall t_{2} \in[0, T] \backslash \mathscr{S}_{D}\left(t_{\text {min }}\right)
$$

In the same way, $u^{*}$ is a solution of the optimization problem in (16) if and only if a similar inequality holds for all $t_{\text {min }} \in \operatorname{supp}\left(f^{\prime}\right)$ :

$$
D_{a, D}\left(t_{1}\right) \leq D_{a, D}\left(t_{2}\right) \forall t_{1} \in \mathscr{S}_{D}\left(t_{\text {min }}\right) \forall t_{2} \in[0, T] \backslash \mathscr{S}_{D}\left(t_{\text {min }}\right)
$$

It is shown now that an alternative formulation of the Nash equilibrium condition (16) can be provided in the variable $q$ when the inflexible demand is broadcast to the devices:

Theorem 1: Consider a profile $D$ which is equal to the inflexible demand $D_{i}$ and fulfils Assumption 2. The equilibrium condition (16) is satisfied for $D=D_{i}$ if and only if:

$$
\Lambda_{f}(q) \leq \Lambda_{D_{i}}(q) \quad \forall q \in\left[q_{\min }, q_{\max }\right]=\operatorname{supp}\left(f^{\prime}\right)
$$

Proof: When $D=D_{i}$, the inflexible demand can be considered as a function $\bar{D}_{i}(q)=Q_{D}^{-1}(q)$ of the measure $q$ since by definition $Q_{D}^{-1}(q)=Q_{D_{i}}^{-1}(q)$ returns the value of $D_{i}$ at a given $q$. As a preliminary result, we show that condition (18) is equivalent to impose that the aggregate demand $\bar{D}_{a, D_{i}}(q)=\bar{D}_{i}(q)+\bar{D}_{f}(q)$ is a nondecreasing function on the support of $f^{\prime}$. If one considers Definition 3 and equation (12), it is straightforward to verify that the left member in (18) corresponds to the derivative of $\bar{D}_{f}(q)$ with changed sign. 
Similarly, the term $\Lambda_{D_{i}}$ in (18) is equal to the derivative of $\bar{D}_{i}$. The inequality (18) can then be written as follows:

$$
\bar{D}_{a, D_{i}}^{\prime}(q)=\bar{D}_{i}^{\prime}(q)+\bar{D}_{f}^{\prime}(q) \geq 0 \quad \forall q \in\left[q_{\min }, q_{\max }\right]
$$

To verify that such condition is necessary and sufficient for a Nash equilibrium when $D_{i}$ is broadcast, the equivalent in time can be considered:

$$
D_{a, D_{i}}\left(t_{1}\right) \leq D_{a, D_{i}}\left(t_{2}\right) \forall\left(t_{1}, t_{2}\right):\left\{\begin{array}{c}
D_{i}\left(t_{1}\right)<D_{i}\left(t_{2}\right) \\
Q_{D_{i}}\left(D_{i}\left(t_{1}\right)\right) \in \operatorname{supp}\left(f^{\prime}\right) \\
Q_{D_{i}}\left(D_{i}\left(t_{2}\right)\right) \in \operatorname{supp}\left(f^{\prime}\right)
\end{array}\right.
$$

In fact, to each $t_{1}$ and $t_{2}$ as specified above can be associated $q_{1}=Q_{D_{i}}\left(D_{i}\left(t_{1}\right)\right)$ and $q_{2}=Q_{D_{i}}\left(D_{i}\left(t_{2}\right)\right)$ which belong to the support of $f^{\prime}$ and with $q_{1}<q_{2}$. From (19) it follows that:

$$
D_{a, D_{i}}\left(t_{1}\right)=\bar{D}_{a, D_{i}}\left(q_{1}\right) \leq \bar{D}_{a, D_{i}}\left(q_{2}\right)=D_{a, D_{i}}\left(t_{2}\right)
$$

Consider now the equilibrium condition presented in (17): taking into account the definition of $\mathscr{S}_{D}$, it can be seen that it must be verified for all $\left(t_{1}, t_{2}\right) \in([0, T] \times[0, T]) \backslash\left(\mathscr{T}_{\min } \cup \mathscr{T}_{\max }\right)$ with $D_{i}\left(t_{1}\right) \leq D_{i}\left(t_{2}\right)$, where $\mathscr{T}_{\min }$ and $\mathscr{T}_{\max }$ are defined as follows:

$$
\mathscr{T}_{\text {min }}:=\left\{\left(t_{1}, t_{2}\right): Q_{D_{i}}\left(D_{i}\left(t_{1}\right)\right)<q_{\min } \wedge Q_{D_{i}}\left(D_{i}\left(t_{2}\right)\right)<q_{\text {min }}\right\}
$$$$
\mathscr{T}_{\text {max }}:=\left\{\left(t_{1}, t_{2}\right): Q_{D_{i}}\left(D_{i}\left(t_{1}\right)\right)>q_{\max } \wedge Q_{D_{i}}\left(D_{i}\left(t_{2}\right)\right)>q_{\max }\right\}
$$

Within the pairs of time instants to be considered, the two cases with respectively $Q_{D_{i}}\left(D_{i}\left(t_{1}\right)\right)<q_{\min }$ and $Q_{D_{i}}\left(D_{i}\left(t_{2}\right)\right)>q_{\max }$ are always verified when (18) holds. For the first case, notice that $K\left(Q_{D_{i}}^{-1}(q)\right)=\bar{D}_{a, D_{i}}(q)$, corresponding to the aggregate demand at $t$ when $Q_{D_{i}}\left(D_{i}(t)\right)=q$, is monotonic increasing from (15) on $\left[Q_{D_{i}}\left(D_{i}\left(t_{1}\right)\right), q_{\min }\right]$ and from (19) on $\left[q_{\min }, Q_{D_{i}}\left(D_{i}\left(t_{2}\right)\right)\right]$. A similar proof can be provided when $Q_{D_{i}}\left(D_{i}\left(t_{2}\right)\right)>q_{\max }$. Condition (17), in the remaining case $q_{\min } \leq Q_{D_{i}}\left(D_{i}\left(t_{1}\right)\right)<Q_{D_{i}}\left(D_{i}\left(t_{2}\right)\right) \leq q_{\max }$, directly corresponds to the time equivalent (20) of (18), concluding the proof.

Having verified under which conditions an equilibrium is obtained by broadcasting the inflexible demand $D_{i}$, we want to understand if there exist other profiles that can be transmitted to the appliances population when the conditions of Theorem 1 do not hold. In this respect, we introduce the class of profiles $\mathscr{D}_{i}$ which induce the same $u^{*}$ as $D_{i}$ (and equal aggregate demand) when they are broadcast. In particular, $D(\cdot)$ belongs to $\mathscr{D}_{i}$ if these conditions are satisfied:

$$
\begin{gathered}
\left\{t: Q_{D}(D(t))<q_{\min }\right\}=\left\{t: Q_{D}\left(D_{i}(t)\right)<q_{\min }\right\} \\
\left\{t: Q_{D}(D(t))>q_{\max }\right\}=\left\{t: Q_{D}\left(D_{i}(t)\right)>q_{\max }\right\} \\
Q_{D}(D(t))=Q_{D_{i}}\left(D_{i}(t)\right) \forall t: Q_{D_{i}}\left(D_{i}(t)\right) \in\left[q_{\min }, q_{\max }\right]
\end{gathered}
$$

It is now possible to provide the following result:

Theorem 2: If (18) does not hold, there exists no broadcast profile $D(\cdot)$ that satisfies Assumption 2 and the equilibrium condition (16).

Proof: It is straightforward to verify the theorem statement for $D(\cdot) \in \mathscr{D}_{i}$ since, by definition, the corresponding $u^{*}$ and aggregate demand are equal to the ones obtained broadcasting $D_{i}$ and therefore the results of Theorem 1 can be extended to the present case. For a profile $D(\cdot) \notin \mathscr{D}_{i}$ it can be shown that, if any condition in (23) is violated, an equilibrium is not achieved. Considering initially that (23a) and (23b) hold while (23c) is violated, we assume there exists $t_{2}$ such that $Q_{D_{i}}\left(D_{i}\left(t_{2}\right)\right) \in\left[q_{\min }, q_{\max }\right]$ and $Q_{D_{i}}\left(D_{i}\left(t_{2}\right)\right)<Q_{D}\left(D\left(t_{2}\right)\right)$ (the proof is similar when the opposite inequality holds). The following sets are introduced:

$$
\begin{aligned}
& \mathscr{T}_{i_{-}}:=\left\{t: q_{\min } \leq Q_{D_{i}}\left(D_{i}(t)\right) \leq Q_{D_{i}}\left(D_{i}\left(t_{2}\right)\right)\right\} \\
& \mathscr{T}_{-}:=\left\{t: q_{\min } \leq Q_{D}(D(t)) \leq Q_{D}\left(D\left(t_{2}\right)\right)\right\}
\end{aligned}
$$

Evaluating the corresponding Lebesgue measures yields:

$$
\mu\left(\mathscr{T}_{i_{-}}\right)=Q_{D_{i}}\left(D_{i}\left(t_{2}\right)\right)-q_{\text {min }}<Q_{D}\left(D\left(t_{2}\right)\right)-q_{\text {min }}=\mu\left(\mathscr{T}_{-}\right)
$$

Since we are assuming that (23a) and (23b) hold, this implies that there exists $t_{1} \in \mathscr{T}_{-} \backslash \mathscr{T}_{i_{-}}$such that:

$$
Q_{D}\left(D\left(t_{1}\right)\right)<Q_{D}\left(D\left(t_{2}\right)\right) \quad Q_{D_{i}}\left(D_{i}\left(t_{1}\right)\right)>Q_{D_{i}}\left(D_{i}\left(t_{2}\right)\right)
$$

or equivalently:

$$
D\left(t_{1}\right)<D\left(t_{2}\right) \quad D_{i}\left(t_{1}\right)>D_{i}\left(t_{2}\right)
$$

Notice now that, if the demand profile $D$ is broadcast, for all $t_{\min } \in\left[Q_{D}\left(t_{1}\right), Q_{D}\left(t_{2}\right)\right)$ it holds:

$$
t_{1} \in \mathscr{S}_{D}\left(t_{\text {min }}\right) \quad t_{2} \notin \mathscr{S}_{D}\left(t_{\text {min }}\right)
$$

This means that $D_{f}\left(t_{1}\right)>D_{f}\left(t_{2}\right)$ and therefore the following holds for the aggregate demand:

$$
D_{i}\left(t_{1}\right)+D_{f}\left(t_{1}\right)>D_{i}\left(t_{2}\right)+D_{f}\left(t_{2}\right)
$$

Consequently, the equilibrium condition (17), equivalent to (16), is violated for $t_{\min } \in\left[Q_{D}\left(t_{1}\right), Q_{D}\left(t_{2}\right)\right)$. The proof is concluded by showing the same results when conditions (23a) or (23b) are violated. In these cases, it is possible to verify that there still exist $t_{1}$ and $t_{2}$ for which (26) holds and a similar procedure can then be followed.

We can finally conclude that, if the inequality (18) is satisfied, an equilibrium can be achieved by broadcasting $D_{i}$ or any equivalent profile which belongs to $\mathscr{D}_{i}$ as defined by (23). In the opposite case, under the current assumptions, it is not possible to achieve an equilibrium. In this respect, it is useful to specify the following:

Remark 3: If Assumption 2 does not hold, the optimal power profile for each appliance is in general not unique. The definitions of $u^{*}$ and $\bar{D}_{f}$ provided in the previous section are no longer valid and the presented equilibrium conditions do not apply. It will be shown in a future work that, in some cases, it is still possible to achieve an equilibrium but an increased level of centralization may be required.

\section{Simulation Results}

The equilibrium conditions presented in the previous section are now tested in simulations. A typical UK demand profile [10] is considered for the function $D_{i}$ of inflexible demand, adopting a time step $\Delta t=0.01 \mathrm{~h}$. If one denotes by $D_{i}^{k}$ the value of inflexible demand at $t=k \cdot \Delta t$, the function 
$Q_{D_{i}}\left(D_{i}^{k}\right)$ is approximated as $\left|\mathscr{S}^{k}\right| \cdot \Delta t$ where $\mathscr{S}^{k}$ is defined as follows:

$$
\mathscr{S}^{k}=\left\{j: D_{i}^{j} \leq D_{i}^{k}\right\}
$$

A case study that satisfies the sufficient condition for equilibrium in Theorem 1 is initially considered. The total energy required by the devices amounts to $55 G W h$ while $f^{\prime}$ is assumed equal to the sum of two truncated gaussians, centered respectively at $t=4.3 \mathrm{~h}$ and $t=8 \mathrm{~h}$. A graphical representation of the equilibrium condition is proposed in Fig. 1 where the negotiable valley capacity $\Lambda_{D_{i}}$ (blue) and the power density of task duration $\Lambda_{f}$ (red) are compared. Note that the inequality (18) is always verified and therefore we expect a Nash Equilibrium in the market when the inflexible demand $D_{i}$ is broadcast. The resulting demand profiles in the variable $q$ are shown in Fig. 2: given the definition of $q$, when $D=D_{i}$ we expect $\bar{D}_{i}(q)$ to be a monotonic increasing function. Conversely, considering that devices will schedule their power consumption at the lowest values of demand, the flexible profile $\bar{D}_{f}(q)$ will always be decreasing. If the equilibrium condition (18) is satisfied, like in this case, the sum $\bar{D}_{a}(q)$ of the two demand components will be a nondecreasing function. Finally, the demand profiles across time are presented in Fig. 3. To show that a Nash equilibrium is achieved, the shaded blue areas represent the scheduled time intervals of power consumption for devices with $t_{\min }$ equal to $3 h, 5 h, 7 h$. One can notice that such intervals correspond to the lowest values of aggregate demand.

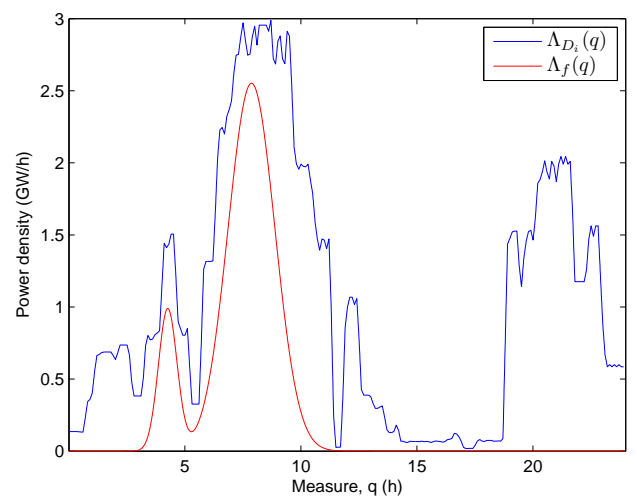

Fig. 1. Graphical representation of the equilibrium condition (18). It can be seen that the power density of task durations $\Lambda_{f}(q)$ (red) is always lesser or equal than the valley capacity $\Lambda_{D_{i}}(q)$ (blue).

We simulate now a scenario for which, under the current assumptions, a Nash equilibrium does not exist. In particular, we consider a population of appliances that require the same amount of total energy (equal to $55 G W h$ ) but we assume in this case that $f^{\prime}$ is equal to a truncated gaussian centered at $t=6 h$. We want to show that, if the power density of task durations $\Lambda_{f}$ for the considered appliances does not fit properly the valley capacity $\Lambda_{D_{i}}$ of the inflexible demand, according to Theorem 1 it is not possible to achieve an equilibrium by broadcasting $D_{i}$. A comparison of $\Lambda_{D_{i}}$ and $\Lambda_{f}$ in this case is provided in Fig. 4: it can be noticed

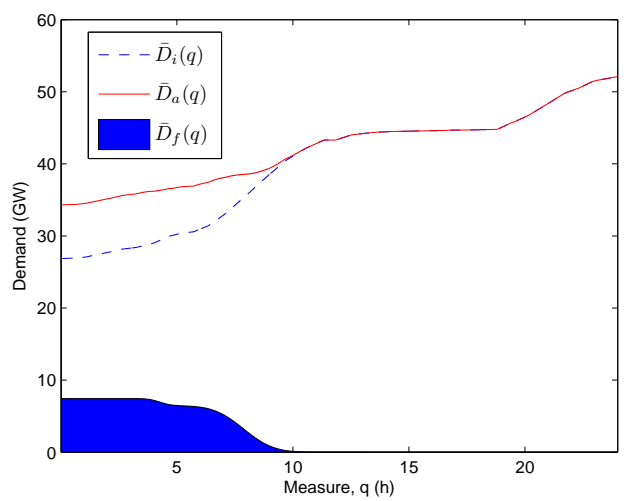

Fig. 2. Inflexible demand $\bar{D}_{i}$ and aggregate demand $\bar{D}_{a}$ as functions of the measure $q=Q_{D_{i}}\left(D_{i}(t)\right)$ when the broadcast $D$ is equal to $D_{i}$. The flexible demand $\bar{D}_{f}$ introduced by the appliances corresponds to the blue area.

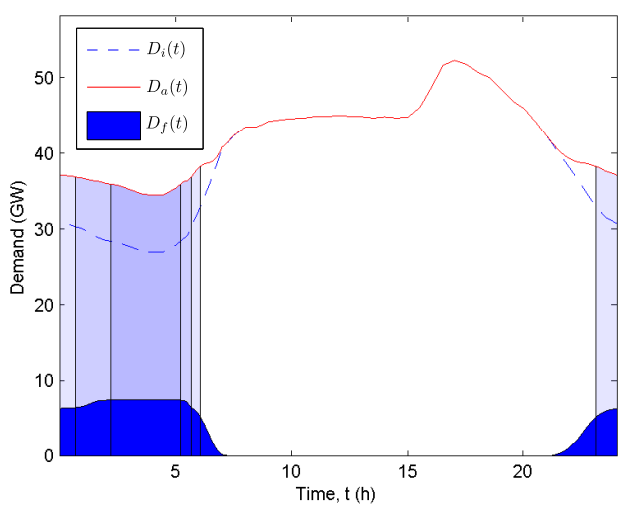

Fig. 3. Inflexible demand $D_{i}$ and aggregate demand $D_{a}$ as functions of time. The blue area represents the flexible demand $D_{f}$ introduced by the appliances population. The shaded blue regions are the time intervals of scheduled power consumption for devices with $t_{\min }$ equal to $3 h, 5 h$ and $7 h$.

that condition (18) is not satisfied. The demand profiles as functions of the measure $q$ when the broadcast demand $D$ is equal to $D_{i}$ are shown in Fig. 5: notice in particular that the monotonicity properties of $\bar{D}_{i}$ and $\bar{D}_{f}$ mentioned in the previous case are still verified. The main difference is that, in the considered scenario, the aggregate demand is not monotonic increasing. To see that an equilibrium is not achieved, one can consider the demand profiles as functions of time shown in Fig. 6, where the shaded blue areas represent, as in the previous case, the time intervals where devices with $t_{\min }$ equal to $3 h, 5 h$ and $7 h$ have scheduled their power consumption. It is straightforward to verify, for example, that the appliances with $t_{\min }$ equal to $3 h$ and $5 h$ could reduce their total cost by shifting part of their power consumption to the small aggregate demand valley which appears around $t=6 h$. The possibility to converge to an equilibrium in multiple steps, with an iterative algorithm, has also been considered. At each iteration the aggregate demand profile obtained at the previous step (starting with $\left.D=D_{i}\right)$ is broadcast to the devices. The results in Fig. 7 show that, as expected from Theorem 2, there is no convergence 
to equilibrium. At each step the appliances change their power profile and the resulting aggregate demand, after some iterations, switches between two distinct functions.

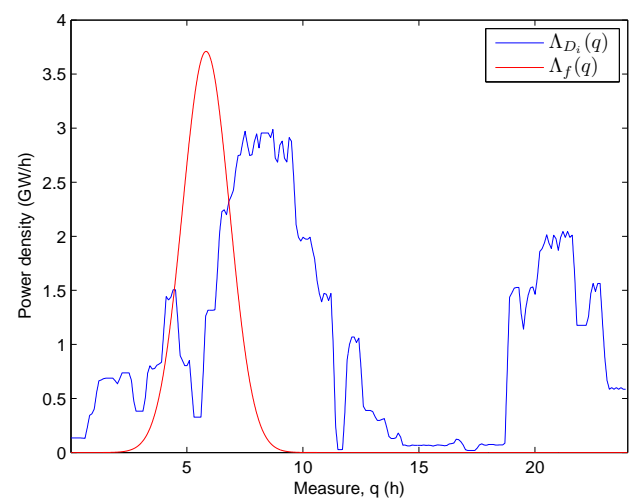

Fig. 4. Graphical representation of the equilibrium condition (18). Notice that in this case such condition is violated in the interval which goes approximatively from $q=4.5 h$ to $q=7 h$.

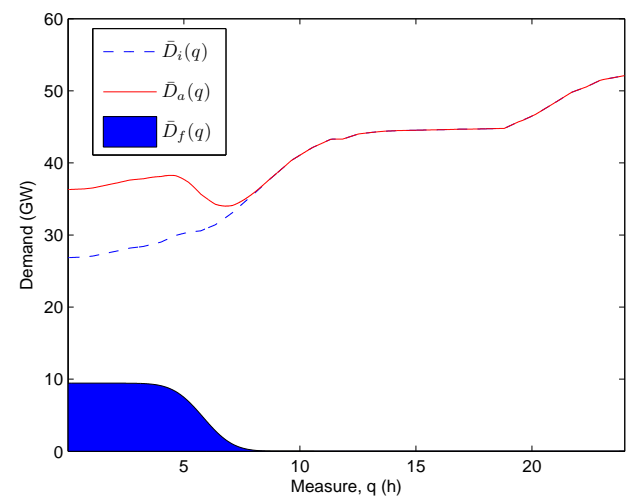

Fig. 5. Inflexible demand $\bar{D}_{i}$ and aggregate demand $\bar{D}_{a}$ as functions of the measure $q=Q_{D_{i}}\left(D_{i}(t)\right)$ when the broadcast $D$ is equal to $D_{i}$. The flexible demand $\bar{D}_{f}$ introduced by the appliances corresponds to the blue area.

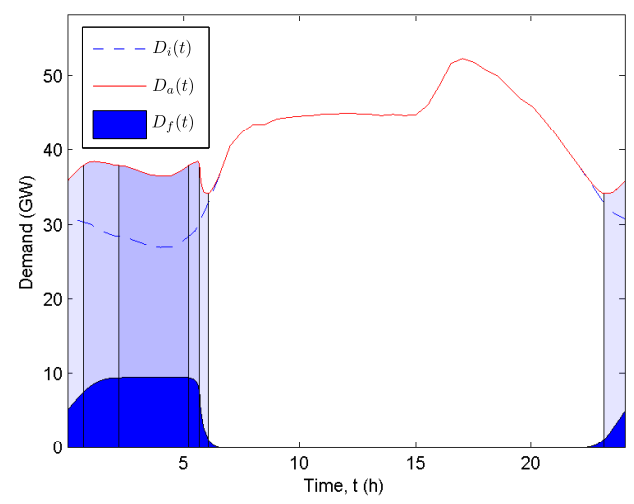

Fig. 6. Inflexible demand $D_{i}$ and aggregate demand $D_{a}$ as functions of time. The blue area represents the flexible demand $D_{f}$ introduced by the appliances population. The shaded blue regions correspond to the time intervals of power consumption of the devices with $t_{\min }$ equal to $3 h, 5 h$ and $7 h$.

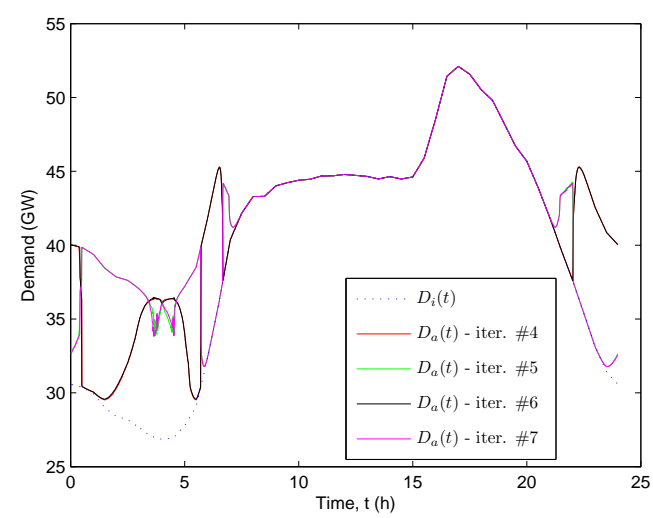

Fig. 7. Inflexible demand $D_{i}$ and aggregate demand $D_{a}$ obtained at certain iterations of the proposed iterative procedure.

\section{CONCLUSIONS}

This paper analyses the integration of a large number of flexible appliances in the energy market, providing necessary and sufficient conditions for the existence of a Nash equilibrium. This is done by approximating the appliances population as a continuum and abstracting its valley-filling capabilities through a power density function. Such quantity is then compared with the negotiable valley capacity, which characterizes the inflexible demand. The methodology, that does not require iterations as it provides convergence to equilibrium in one step, is finally tested in simulations.

\section{REFERENCES}

[1] G. Strbac, "Demand side management: Benefits and challenges," Energy Policy, vol. 36, no. 12, pp. 4419-4426, 2008.

[2] M. Albadi and E. El-Saadany, "A summary of demand response in electricity markets," Electric Power Systems Research, vol. 78, no. 11, pp. 1989-1996, 2008.

[3] C. L. Su and D. Kirschen, "Quantifying the effect of demand response on electricity markets," IEEE Transactions on Power System, vol. 24, no. 3, pp. 1199-1207, 2009.

[4] P. Samadi, H. Mohsenian-Rad, R. Schober, and V. W. S. Wong, "Advanced demand side management for the future smart grid using mechanism design," IEEE Transactions on Smart Grid, vol. 3, no. 3, pp. 1170-1180, 2012.

[5] Z. Fan, "A distributed demand response algorithm and its application to PHEV charging in smart grids," IEEE Transactions on Smart Grid, vol. 3, no. 3, pp. 1280-1290, 2012.

[6] D. Papadaskalopoulos and G. Strbac, "Decentralized participation of flexible demand in electricity markets - part I: Market mechanism," IEEE Transactions on Power Systems, vol. 28, no. 4, pp. 3658-3666, 2013.

[7] H. Mohsenian-Rad, V. W. S. Wong, J. Jatskevich, R. Schober, and A. Leon-Garcia, "Autonomous demand-side management based on game-theoretic energy consumption scheduling for the future smart grid," IEEE Transactions on Smart Grid, vol. 1, no. 3, pp. 320-331, 2010.

[8] A. C. Kizilkale, S. Mannor, and P. E. Caines, "Large scale real-time bidding in the smart grid: A mean field framework," in Proceedings of the 51st IEEE Conference on Decision and Control, 2012, pp. 3680 $-3687$.

[9] L. Gan, U. Topcu, and S. H. Low, "Optimal decentralized protocol for electric vehicle charging," IEEE Transactions on Power Systems, vol. 28, no. 2, pp. 940-951, 2013.

[10] National Grid. Metered half-hourly electricity demands. [Online]. Available: http://www.nationalgrid.com/uk/Electricity/ Data/Demand+Data/ 